\title{
Estimación de calidad del cuidado de enfermería en pacientes sometidos a hemodiálisis
}

\author{
Estimation of quality of nursing care in patients subjected to hemodialysis
}

\section{Estimativa da qualidade do cuidado de enfermagem em pacientes submetidos a hemodiálise}

\author{
Victor Alfonso López Soracipa* \\ Eilyn Alexandra España Barrios** \\ Ana Elena Hernández Garcés ${ }^{* * *}$
}

\section{Resumen}

Objetivo: Evaluar la calidad del cuidado de enfermería en una población de pacientes con tratamiento de hemodiálisis en la ciudad de Bogotá. Materiales y Métodos: Se realizó un estudio descriptivo de corte transversal, mediante la aplicación de la adaptación colombiana del instrumento Care-Q que relaciona actitudes y actividades de cuidado que realiza el enfermero(a) y que fueron evaluadas por el paciente a través de una escala Likert. El universo estuvo conformado por la totalidad de los pacientes en hemodiálisis que pertenecen a un grupo de apoyo de pacientes crónicos, se llegó a un tope de 100 pacientes convocados a los cuales se les aplicó el instrumento. Resultados: Se determinó que entre el $60 \%$ y $85 \%$ de la población evaluaba de manera positiva los cuidados proporcionados por el personal de enfermería. Conclusiones: Se encontró un porcentaje considerable de satisfacción dentro de la población participante, lo cual se contrasta con lo evidenciado en la literatura donde se plantea que el enfermero(a) es el profesional más indicado para el cuidado de este tipo de pacientes. También se hizo evidente la necesidad de generar investigaciones dirigidas a la evaluación continua de la atención de enfermería, debido a que ello facilita la creación de acciones de mejoramiento en la gestión del cuidado y permite trabajar por el cumplimiento de la calidad en la atención en salud.

Palabras clave: Satisfacción del Paciente, Mecanismos de Evaluación de la Atención de Salud, Fallo Renal Crónico

\section{Abstract}

Objective: To evaluate the quality of nursing care in a population of patients with hemodialysis treatment in the city of Bogotá. Materials and Methods: A cross-sectional descriptive study was carried out, through the application of the Colombian adaptation of the Care-Q instrument that relates attitudes and nursing care activities that were evaluated by the patient through a Likert scale. The universe consisted of all the patients on hemodialysis who belong to a support group of chronic patients, reaching a limit of 100 patients summoned to whom the instrument was applied. Results: It was determined that between $60 \%$ and $85 \%$ of the population evaluated in a positive way the care provided by the nursing staff. Conclusions: A considerable percentage of satisfaction was found within the participating population, which is contrasted with what is evidenced in the literature where it is stated that the nurse is the most suitable professional for the care of this type of patients. It made evident the need to generate research aimed at the continuous evaluation of nursing care, because it facilitates the creation of actions for improvement in the management of care and allows working for the fulfillment of quality in health care.

Key words: Patient Satisfaction, Health Care Evaluation Mechanisms, Kidney Failure Chronic

\section{Para citar este artículo/ To reference this article / Para citar este artigo/}

López Soracipa VA, España Barrios EA, Hernández Garcés AE. Estimación de calidad del cuidado de enfermería en pacientes sometidos a hemodiálisis. Rev. cienc. cuidad. 2018;15(1): 71-82

Este es un artículo bajo la licencia CC BY (https://creativecommons.org/licenses/by/4.0/) @ () 
ISSN-PRINT

1794-9831

E-ISSN 2322-7028

Vol. 15 No. 1

Ene - Jun 2018

Cúcuta, Colombia

\section{Resumo}

Objetivo: Avaliar a qualidade do cuidado de enfermagem numa população de pacientes com tratamento de hemodiálise na cidade de Bogotá, Colômbia. Materiais e Métodos: Realizou-se um estudo descritivo de corte transversal, através da aplicação da adaptação colombiana do instrumento Care-Q que relaciona atitudes e atividades de cuidado que realiza o enfermeiro (a) e que foram avaliadas pelo paciente através de uma escala Likert. A amostra esteve conformada pela totalidade dos pacientes em hemodiálise que pertencem a um grupo de apoio de pacientes crônicos, chegouse a um limite de 100 pacientes convocados nos quais o instrumento foi aplicado. Resultados: Determinou-se que entre $60 \%$ e $85 \%$ da população avalia positivamente os cuidados prestados pela equipe de enfermagem. Conclusões: Encontrou-se uma porcentagem considerável de satisfação dentro da população participante, o qual se contrasta com o evidenciado na literatura onde afirma-se que o enfermeiro (a) é o professional mais indicado para o cuidado deste tipo de pacientes. Também se tornou evidente a necessidade de gerar pesquisas dirigidas à avaliação contínua dos cuidados de enfermagem, já que facilita a criação de ações de melhoramento na gestão do cuidado e permite trabalhar pelo cumprimento da qualidade no atendimento em saúde.

Palavras-chave: Insuficiência renal crônica, mecanismos de avaliação do atendimento de saúde, satisfação do paciente

\section{Introducción}

Históricamente se ha trabajado sobre calidad en diferentes industrias; en el sector comercial, el sector automotriz y el textil, entre otros, lo cual ha permitido que todas estas áreas mejoren sus productos, procesos y estándares (1). El sector salud no es ajeno a este tema, por lo cual cada día se hace más relevante para las organizaciones el estudio de la calidad de atención, ya que permite establecer un diagnóstico que facilita el planteamiento de acciones de mejora encaminadas a optimizar los cuidados que se brindan a los pacientes.

La satisfacción generada con la atención al acudir a los servicios de salud se convierte en uno de los indicadores de mayor relevancia para evaluar la calidad. Donabedian (2-3) afirma que la "calidad de la atención es aquella que se espera que pueda proporcionar al usuario el máximo y más completo bienestar después de valorar el balance de ganancias y pérdidas que pueden acompañar al proceso en todas sus partes". En este contexto, se presenta la calidad del cuidado de enfermería como un medio para trabajar por el bienestar de este usuario, en el que sea tratado como un ser integral que requiere satisfacer todas sus necesidades. Dicha satisfacción es proporcionada por el enfermero(a), mediante la utilización de sus conocimientos científicos y de su habilidad interpersonal al interactuar con el individuo.

El personal de enfermería, dentro del sistema de salud, se convierte en un actor importante en el proceso de atención, siendo el acto de cuidado el objetivo principal de esta disciplina, como lo menciona Leininger (4), quien lo cataloga bajo la categoría de necesidad esencial. En este entorno se encuentran los pacientes con enfermedad renal crónica, quienes requieren cuidados integrales y de calidad que faciliten el proceso de aceptación, adaptación y adherencia al tratamiento (5).

La enfermedad renal crónica es considerada como enfermedad de alto costo puesto que genera gastos exorbitantes por el tratamiento que requiere. Se evidencia que más de un millón de personas se mantiene con tratamiento de hemodiálisis $\mathrm{y}$, anualmente, cerca de 200.000 personas inician este tratamiento en el mundo (6).

Por otra parte, diversos estudios (7-8) muestran y ejemplifican el costo elevado que genera la enfermedad renal crónica. Una investigación realizada por la Universidad de Antioquia, en Colombia, encontró que esta patología genera gastos exorbitantes para el sistema general de seguridad social en salud, entidades promotoras de salud y demás prestadores del servicio, debido a los costos en equipos médicos, infraestructura y todo el material necesario para brindar la atención (9).

De acuerdo con el Fondo Colombiano de Enfermedades de Alto Costo, para el periodo 2015-2016, en el país, al menos 359.222 personas fueron diagnosticadas con enfermedad renal crónica, de las cuales el $60 \%$ eran mujeres. El promedio de edad fue de 63 años, 
siendo el $61 \%$ de la población mayor de 60 años y el $29 \%$ personas con edades entre 45 y 59 años. Para este mismo periodo, la prevalencia fue de $61.62 \mathrm{x}$ cada 100.000 habitantes, con una incidencia de 11.01 pacientes x cada 100.000 afiliados y una mortalidad de 28.19 pacientes x cada 100.000 afiliados (10).

La enfermedad renal crónica se convierte en una condición que involucra de manera global la vida de quienes la padecen, generando afecciones físicas, sociales y emocionales. Debido al deterioro secundario durante el curso normal de la patología y el tratamiento, los pacientes disminuyen su productividad, padecen un cambio en la dinámica familiar, ven afectada su autoestima e imagen corporal y se ven obligados a desertar de sus labores académicas o laborales, lo cual no sólo tiene un impacto directo en su calidad de vida, sino que también lo tiene en la de su familia y la red directa de apoyo, ya que son sometidos a estrictos tratamientos sustitutivos como la hemodiálisis.

En este contexto, el personal de enfermería establece prioridades para proporcionar los cuidados y así garantizar la calidad de los mismos. Sumado a esto, el enfermero(a) es el profesional encargado de la atención directa del paciente y es quien planea los cuidados característicos según las necesidades individuales, incluyendo la atención de las manifestaciones físicas, psicológicas y emocionales de la enfermedad (11-12).

De acuerdo con Ortíz, "los usuarios constituyen la razón de ser, no sólo de los profesionales de enfermería, sino también de la empresa que ofrece satisfacción a las necesidades relacionadas con la salud". Este autor también conceptualiza sobre los "momentos de verdad", es decir, aquellos que acontecen cuando el paciente refleja la calidad de la relación con la persona que brinda el servicio y expresa abiertamente a otras personas el agrado o desagrado del satisfactor proporcionado (13).

Son estos "momentos de verdad" los que permiten evaluarel grado de satisfacción con el cuidadobrindado por el enfermero(a). Dentro de la investigación que se planteó, se trabajaron seis dimensiones que tienen que ver con el desempeño en actividades propias del cuidado de Enfermería: determinación de necesidades, comunicación, empatía, confianza, planeación y seguimiento- monitoreo. Para cada una se evaluó el grado de satisfacción percibido por el paciente en hemodiálisis y esto se tomó como indicador de la calidad del cuidado brindado por el enfermero(a).

De acuerdo con el contexto planteado, se hace necesaria la evaluación de la labor del profesional de enfermería con pacientes en tratamiento de hemodiálisis, puesto que permite catalogar el impacto de sus intervenciones dentro de la población de enfermos crónicos. Así mismo, se hace evidente que la caracterización por niveles de satisfacción generados con la atención es un indicador directo de la calidad de las organizaciones que prestan servicios de salud, lo cual permite que dichas organizaciones se enfoquen en la mejora permanente, con un objetivo único: el bienestar del paciente.

\section{Objetivos}

\section{Objetivo General}

Evaluar la calidad del cuidado de enfermería en una población de pacientes con tratamiento de hemodiálisis en la ciudad de Bogotá, mediante la aplicación de instrumento Care-Q en el primer semestre del año 2017.

\section{Objetivos Específicos}

- Describir, de manera general, las características demográficas de la población objeto de estudio.

- Caracterizar el nivel de satisfacción de los pacientes con tratamiento de hemodiálisis.

- Describir la calidad del cuidado de enfermería encontrada en los pacientes con hemodiálisis en las subescalas de Accesibilidad, Explica y facilita, Conforta, Se anticipa, Mantiene relación de confianza, Monitorea y hace seguimiento, en términos del instrumento Care-Q de Patricia Larson.

\section{Materiales y métodos}

Se trata de un estudio descriptivo de corte transversal, cuya población objeto estuvo constituida por hombres y mujeres mayores de dieciocho años, que se encontraban en tratamiento de hemodiálisis y que formaban parte de un grupo de apoyo de pacientes crónicos de la ciudad de Bogotá durante el primer semestre de 2017. 
ISSN-PRINT

1794-9831

E-ISSN 2322-7028

Vol. 15 No. 1

Ene - Jun 2018

Cúcuta, Colombia
Todos los pacientes fueron convocados a través de un integrante del grupo de apoyo que se reúne para otro tipo de actividades y encuentros diferentes a las sesiones de hemodiálisis, razón por la cual el estudio se llevó a cabo fuera de las instituciones de salud donde reciben tratamiento. Se les explicó la intención y objetivos del estudio y los participantes aceptaron su inclusión en la investigación mediante la firma del consentimiento informado.

\section{Criterios de inclusión para la aplicación del estudio:}

- Pacientes que estuvieran en tratamiento de hemodiálisis durante el primer semestre del año 2017.

- Pacientes que no presentaran alteraciones mentales o algún estado de discapacidad que le impidiera responder al instrumento.

- Pacientes que voluntariamente desearan responder el instrumento, por medio de consentimiento informado.

\section{Criterios de exclusión:}

- Personas que presentaran alteraciones neurológicas, mentales o psiquiátricas que le impidan responder la prueba.

\section{Muestra:}

Se realizó el cálculo de tamaño de muestra utilizando el software epidemiológico Epidat versión 4.1, bajo los siguientes parámetros: un intervalo de confianza del $95 \%$, un error alfa del $5 \%$ y una proporción desconocida del $50 \%$, donde, para la totalidad de los pacientes del grupo de apoyo -239-, se calculó una muestra de 132 participantes y se llegó a un tope de 100 pacientes convocados a quienes se les aplicó el instrumento. Se decidió trabajar con este población dado que se considera que este valor es representativo para la población total que conforma el grupo de apoyo de pacientes crónicos, por lo cual, para el caso del presente estudio, se llevó a cabo un muestro no probabilístico por conveniencia.

El instrumento seleccionado para evaluar la calidad del cuidado de enfermería se denomina Caring Assessment Instrument (Care-Q, por sus siglas en inglés), que fue realizado en 1981 y validado por Larson (14), enfermera con amplia experiencia en investigación en percepción del cuidado de enfermería, quien realizó sus estudios de doctorado relacionados con la percepción del cuidado de pacientes con cáncer por enfermeras oncólogas.

Care-Q está compuesto por 50 ítems divididos en seis subescalas para evaluar la satisfacción de la calidad del cuidado y los comportamientos o conductas del personal de enfermería al proporcionar este cuidado. Califica de 1 a 5 cada ítem, siendo 1 la más baja calificación o menos importante donde la enfermera no lo practica o no lo tiene en cuenta al brindar el cuidado y 5 la más alta calificación o la más importante teniendo en cuenta que la enfermera cumple con estas conductas para la satisfacción del paciente. El instrumento plantea una evaluación de cada ítem, permitiéndole al usuario expresar su percepción de calidad del cuidado con relación a la interacción que se establece entre el personal de enfermería al requerir un servicio de salud.

Las subescalas son:

1. Accesible: contenida por 6 preguntas que consisten en evaluar aspectos relacionados con la relación que establece la enfermera con el paciente para ofrecer cuidados de manera oportuna y que se prevean complicaciones.

2. Explica y facilita: contiene 6 preguntas relacionadas con las actividades de educación al paciente en cuanto a la enfermedad, su tratamiento y rehabilitación, además de si estas actividades son comprensibles o no por el mismo.

3. Conforta: compuesta por 9 preguntas que se refieren a los cuidados proporcionados con el fin de que el paciente se sienta cómodo e incentivado para el afrontamiento del proceso que implica la enfermedad.

4. Se anticipa: contiene 11 preguntas que hacen referencia a si los profesionales de enfermería ejecutan la debida planeación de actividades terapéuticas teniendo en cuenta las necesidades individualizadas de cada paciente y así prevenir complicaciones.

5. Mantiene relación de confianza: integrada por 10 preguntas en torno al establecimiento, por parte de los enfermeros, de una relación asertiva y empática con los pacientes. Este ítem está relacionado con la cercanía y cuidados directos, haciéndolos sentir importantes para el proceso de atención de enfermería. 
6. Monitorea y hace seguimiento: contiene 8 preguntas que permiten establecer el soporte que el enfermero(a) proporciona a los cuidados a través del conocimiento científico y la aplicación del proceso de atención de enfermería.

El instrumento fue traducido, validado y adaptado en el año 2009 para la población colombiana, en el estudio piloto de validación del cuestionario Care-Q realizado por Sepúlveda (15), en donde mostró una varianza de $52 \%$ y un alfa de Cronbach de 0.868. El análisis de validez por dimensión fue: Accesible, $\alpha=0.627$; Explica y facilita, $\alpha=0.57$; Conforta, $\alpha=0.79$; Se anticipa, $\alpha=0.566$; Mantiene relación de confianza, $\alpha=0.591$; Monitorea y hace seguimiento, $\alpha=0.907$. Se obtuvo como resultado una versión corta del instrumento que se adapta a las características propias de la población adulta colombiana, eliminando algunos ítems y mejorando su relación conceptual y estadística. Finalmente, el instrumento Care-Q para población colombiana quedó conformado por 46 preguntas.

Se realizó una prueba piloto en donde se identificó que cada ítem del instrumento era claro y comprensible y se determinó un tiempo promedio de 30 minutos de respuesta por cada participante. Una vez fueron seleccionados los pacientes que cumplieron con los criterios de inclusión, se realizó la aplicación del instrumento en un lugar externo a las instituciones de salud.

El procesamiento de los datos se ejecutó por medio de una base de Excel 2016, en donde se efectuó una categorización con el número de mujeres y hombres participantes en el estudio. Así mismo, se realizó la clasificación por edades y estado de afiliación al sistema de salud.

Por otro lado, el análisis de los datos se realizó partiendo de los resultados globales de cada una de las seis subescalas y, posteriormente, se revisaron estos resultados con los porcentajes encontrados en cada una de las preguntas que integraban la subescala. De este análisis se resaltaron los hallazgos más relevantes.

La investigación fue revisada y aprobada por el Comité Académico de la Facultad de Enfermería de la Universidad Antonio Nariño. Para la ejecución de la investigación se realizó y aplicó un consentimiento informado, teniendo en cuenta los parámetros planteados en el artículo 15 de la Resolución 8430 de1993. Se informó a quienes deseaban participar el nombre, tipo y finalidad del estudio, así como la identidad de los responsables de la investigación, especificando, además, que la participación era voluntaria y que no causaría daño alguno como persona ni como paciente, puesto que se guardaría confidencialidad y privacidad de la información obtenida por medio de la aplicación del instrumento.

\section{Resultados}

Dentro de las características de la población se encontró que el $59 \%$ hombres y el $41 \%$ eran mujeres, que el promedio de edad de los participantes fue de 23 a 82 años, que el $22 \%$ de los pacientes laboraban de forma independiente, lo cual permitió evidenciar que, a pesar de las sesiones de hemodiálisis, estos pacientes lograban mantener un curso normal en sus actividades diarias (Ver Tabla 1). Como hallazgo relevante se encontró que un bajo porcentaje de la población no contaba con ningún tipo de estudio, pero sabían leer y escribir.
ISSN-PRINT

1794-9831

E-ISSN 2322-7028

Vol. 15 No. 1

Ene - Jun 2018

Cúcuta, Colombia 
ISSN-PRINT

1794-9831

E-ISSN 2322-7028

Vol. 15 No. 1

Ene - Jun 2018

Cúcuta, Colombia

Tabla 1. Caracterización demográfica población del estudio

\begin{tabular}{|c|c|c|}
\hline \multirow{3}{*}{$\begin{array}{c}\text { Estrato } \\
\text { socio económico }\end{array}$} & 1 & $15 \%$ \\
\hline & 2 & $57 \%$ \\
\hline & 3 & $28 \%$ \\
\hline \multirow{5}{*}{$\begin{array}{c}\text { Entidad Promotora de } \\
\text { Salud }\end{array}$} & Capital salud & $35 \%$ \\
\hline & $\mathrm{CCF}$ & $22 \%$ \\
\hline & Cafesalud & $32 \%$ \\
\hline & CCF Boyacá & $8 \%$ \\
\hline & Colsubsidio & $3 \%$ \\
\hline \multirow{6}{*}{ Ocupación } & Pensionado & $20 \%$ \\
\hline & Desempleado & $6 \%$ \\
\hline & Empleado & $31 \%$ \\
\hline & Hogar & $20 \%$ \\
\hline & Estudiante & $1 \%$ \\
\hline & Trabajador independiente & $22 \%$ \\
\hline \multirow{5}{*}{ Estado civil } & Soltero & $2 \%$ \\
\hline & Casado & $72 \%$ \\
\hline & Viudo & $2 \%$ \\
\hline & Separado & $7 \%$ \\
\hline & Unión libre & $17 \%$ \\
\hline \multirow{5}{*}{ Formación } & Sin estudios & $8 \%$ \\
\hline & Primaria & $22 \%$ \\
\hline & Bachillerato & $30 \%$ \\
\hline & Técnico & $33 \%$ \\
\hline & Universitario & $7 \%$ \\
\hline
\end{tabular}

Fuente: Datos encabezado consentimiento informado, 2017

De acuerdo con las 6 subescalas del instrumento, se encontró que:

En la subescala Accesibilidad, el $67 \%$ de la población manifestó sentirse satisfecha en cuanto a si el enfermero(a) ofreció al paciente medidas que aliviaran el dolor y si el profesional se acercaba para la realización de procedimientos. Apenas el $2 \%$ de la población manifestó sentirse muy poco satisfecha respecto de la información que brinda el enfermero(a) sobre grupos de ayuda para el control y seguimiento de su enfermedad. También se encontró que el $2 \%$ de la población percibe que los medicamentos no se administran a tiempo, los procedimientos no se realizan de manera oportuna y que no se atiende al llamado de manera rápida, convirtiéndose estas situaciones en generadores de insatisfacción en la categoría de Accesibilidad.
En lo concerniente a la subescala Explica y facilita, el $61 \%$ de la población manifestó sentirse muy satisfecha. Dentro de esta subescala, el $29 \%$ manifestó sentirse satisfecho con interrogantes como: ¿El enfermero(a) le sugiere preguntas para que usted le formule a su doctor cuando lo necesite? y ¿El enfermero(a) es honesto(a) en cuanto a su condición médica? En gran parte de las preguntas de esta subescala, la población refirió sentirse poco satisfecha, ya que percibió recibir poca educación acerca de su autocuidado. Por otro lado, un $62 \%$ de la población manifestó sentirse muy satisfecha con respecto a las explicaciones y facilidad para comprender la información brindada por el enfermero(a), atribuido esto, principalmente, a que el profesional conoce las características demográficas y de formación académica de la población que atiende.

En la subescala Conforta, el $56 \%$ de la población 
refirió sentirse muy satisfecha, lo cual se contrapone con la pregunta donde se interrogó acerca de la atención que presta el enfermero(a) en las horas de la noche, la cual presentó una valoración de muy poco satisfecho $(59 \%)$ manifestado principalmente por "ausencia" del personal de enfermería cuando es requerido por el paciente. En cuanto a la pregunta que evaluó la amabilidad y alegría del enfermero(a) durante las intervenciones, se observó un $56 \%$ de satisfacción.

En la subescala Se anticipa, entre un $25 \%$ y $30 \%$ de la población manifestó sentirse satisfecha. De otro lado, con preguntas como: ¿El enfermero(a) está pendiente de sus necesidades para prevenir posibles alteraciones en su plan de intervención?, apenas el 2 $\%$ de la población encuestada manifestó sentirse poco satisfecha.

En la subescala denominada Mantiene relación de confianza se observó un predominio en la manifestación de sentirse muy satisfecho, con un 63 $\%$. Esta subescala incluía preguntas relacionadas con el porte del carné de identificación y el uniforme que caracteriza al profesional, así como la percepción que tiene el paciente sobre la organización del enfermero(a) en la realización de su trabajo.

Por último, la subescala Monitorea y hace seguimiento fue la que presentó mayor porcentaje de satisfacción, con un $84 \%$, ya que los pacientes percibieron que los cuidados físicos, la educación a la familia y la seguridad en la atención son las principales actividades que favorecen un mayor estado de satisfacción.

En términos generales, se observó, en la valoración comparativa global de las seis subescalas, que la subescala Se anticipa es la que presentó mayor grado de valoración como muy poco satisfecho, dado que los pacientes no perciben que sean incluidos en la creación de los planes de cuidado, ni que se busquen los momentos adecuados para hablar con ellos y su familia, generando un estado de insatisfacción al sentirse -de cierta manera- ignorados y poco comprendidos por el profesional de enfermería. (Ver Gráfica 1).

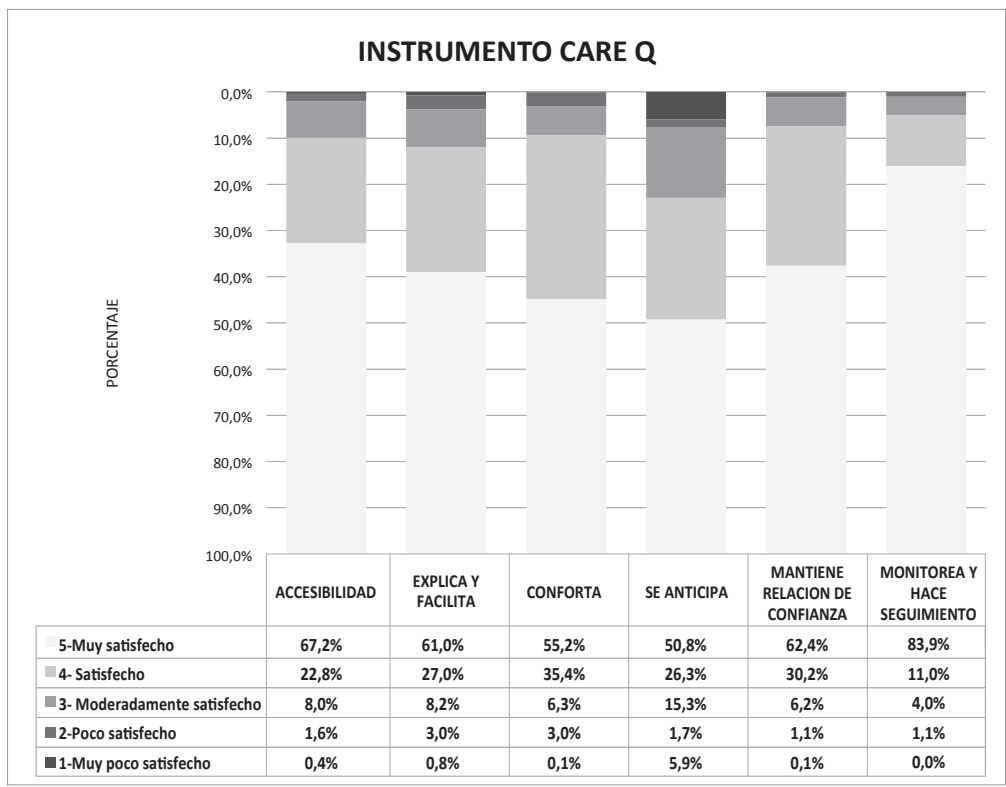

Gráfica 1. Subescalas Instrumento Care-Q

Fuente: Instrumento de evaluación Care-Q, 2017 
ISSN-PRINT

1794-9831

E-ISSN 2322-7028

Vol. 15 No. 1

Ene - Jun 2018

Cúcuta, Colombia

\section{Discusión}

De la información obtenida con la aplicación del instrumento se evidenció que, para la totalidad de la población del estudio, entre el $60 \%$ y $85 \%$ manifestó sentirse muy satisfecha con las actividades relacionadas con el cuidado de enfermería, incluyendo cada una de las temáticas manejadas en las seis subescalas evaluadas. En este contexto se observa actualmente una necesidad sentida de investigar el impacto que tiene la satisfacción del paciente dentro de la calidad de la atención (16) y en fenómenos como la adherencia al tratamiento, la disminución de los costos de atención y el impacto de los eventos adversos (17).

Existen varios estudios en diferentes países y organizaciones de salud (18-23) que ratifican la importancia de medir la satisfacción del paciente con la atención recibida, como un indicador de la calidad de la prestación del servicio y como una herramienta para enfocar planes de intervención y mejoramiento.

Como lo afirma Noest etal. (24), el número de pacientes con necesidades complejas en salud está aumentando $\mathrm{y}$, por lo tanto, se necesitan urgentemente iniciativas para ampliar el aseguramiento de la calidad a través de las fronteras organizacionales y los sectores sanitarios. Como lo describe Machuca (25), es el momento de preguntarse si el paciente encuentra, en la atención de enfermería, la satisfacción de sus necesidades no sólo físicas sino emocionales y si encuentra en el enfermero(a) la confianza suficiente para obtener la transformación que requiere para mejorar su calidad de vida. Esta autora también afirma que la calidad de la atención se refleja en la satisfacción del paciente, pero ésta también se refleja en la satisfacción del personal de enfermería que presta el servicio.

La enfermedad renal crónica supone un cambio no solamente en las condiciones físicas y de salud del paciente; tiene un impacto directo en todas sus dimensiones: social, familiar, comunicativa, de pareja, espiritual y ético-moral, entre otras. Muchos de estos pacientes se ven enfrentados de manera súbita a un tratamiento que condiciona la oportunidad de vivir, por lo cual atraviesan por una reestructuración de dichas dimensiones y es allí donde el enfermero(a) juega un papel indispensable como soporte en este periodo de transición y adaptación a estos cambios, no sólo desde el punto de vista del tratamiento de hemodiálisis en sí, sino también desde el significado que tiene la enfermedad para el paciente y como él concibe su vida con esta nueva condición crónica de salud.

Es por ello que, como lo relata Moreno (26), para los enfermeros(as) es fundamental entender el fenómeno de la cronicidad, puesto que ellos se convierten en el principal grupo de apoyo de este tipo de pacientes y de sus familiares y/o cuidadores. Así también, como lo señala Barrera et al. (27), es en este entorno en donde la intervención de enfermería debe estar enfocada hacia el manejo completo de la enfermedad, conociendo sus riesgos, complicaciones y secuelas, así como las estrategias de tratamiento y de apoyo para la familia y el cuidador.

Lo anterior se hizo evidente en esta investigación, puesto que los resultados se asemejan con varios estudios que han utilizado metodologías similares y el instrumento Care-Q en diferentes escenarios y/o servicios de salud. Estudios como el realizado por Cortés y Serrezuela (28) en un servicio de urgencias de la ciudad de Bogotá D.C, encontró que el nivel de satisfacción sobre la atención de enfermería es bueno, con porcentajes de $43 \%$ con alto grado, 42 $\%$ con satisfacción media y un $15 \%$ con un nivel bajo, concluyendo que, si bien el nivel de satisfacción está en la escala favorable, se evidencia una clara insatisfacción con relación a la comunicación entre el personal de enfermería y el paciente en torno a su situación de salud, hallazgos similares a los encontrados en el presente estudio, donde en la subescala Explica y facilita la población manifestó sentirse poco satisfecha debido a la escasa educación sobre sus cuidados y anticipación sobre los mismos para que resulten oportunos y eficientes.

Así mismo, producto de la aplicación del instrumento en dos instituciones de salud de la costa atlántica colombiana (29), se logró establecer que las subescalas con menor grado de satisfacción fueron Se anticipa y Explica y facilita, resultados que permitieron a los investigadores recomendar hacer mayor énfasis en los ítems "La enfermera le sugiere preguntas que usted puede formularle a su doctor cuando lo necesite" y "La enfermera busca la oportunidad más adecuada para hablar con usted y su familia sobre su situación de salud".

De otro lado, la investigación de Molina (30) concluyó que, al aplicar el instrumento en la atención de enfermería domiciliaria, las subescalas con mayor 
porcentaje de satisfacción fueron Conforta y Monitorea y hace seguimiento, con un porcentaje alrededor del 90 $\%$, resultados muy parecidos a los de la investigación con los pacientes en hemodiálisis donde la subescala Monitorea y hace seguimiento presentó nivel de muy satisfecho -por encima del $80 \%$-, relacionado directamente con los cuidados proporcionados, con el manejo del conocimiento científico, la aplicación de las etapas del proceso de atención de enfermería y la seguridad que percibe el paciente por parte del enfermero(a) al momento de realizar una intervención.

El estudio realizado por Bautista (31) en una institución pública de salud de Cúcuta, utilizando el instrumento Care-Q, concluyó que "el conocer la percepción de los usuarios permite evaluar la calidad de los cuidados ofrecidos en los servicios de salud, para ser más competitivos y satisfacer las necesidades y expectativas de cuidado del paciente hospitalizado en el servicio de clínicas médicas", premisa muy similar a la manejada en esta investigación, ya que se pudo identificar que la satisfacción que tiene el paciente con la atención que recibe del personal de salud es un indicador directo de la calidad de las instituciones. De igual forma, se evidenció que un paciente que se sienta "bien atendido", se siente a su vez más identificado con el personal que lo maneja $\mathrm{y}$, de esta manera, se ve más motivado a participar activamente en su tratamiento y en el mantenimiento de su salud. Podría decirse, entonces, que la satisfacción percibida por parte del paciente acerca de los cuidados recibidos es directamente proporcional al compromiso con su salud, el manejo de su enfermedad y la adherencia al tratamiento.

Por esto, como lo exponen Appleby y Camacho (32), "es necesario plantear las posibles líneas estratégicas en términos globales de salud y calidad de vida de los pacientes con procesos crónicos, la mejora de la calidad de los servicios y costo-efectividad de las intervenciones, una mayor cohesión de los equipos asistenciales y, por supuesto, que todo ello contribuya a la sostenibilidad del sistema sanitario y a la satisfacción de los usuarios".

Se hace evidente, entonces, que enfatizar en prácticas como el ofrecimiento de unidades adaptadas 0 ajustadas para la comodidad del paciente, la interacción en la realización de los cuidados y la empatía en la aplicación de los mismos, resultan de vital importancia, son inherentes al papel de enfermería y no deben quedar en segundo plano en ningún momento, ya que hacen parte de la atención integral y permiten evaluar constantemente la calidad del cuidado.

Los pacientes con tratamiento de hemodiálisis son individuos que requieren una atención continua e individualizada por parte del personal de enfermería; por consiguiente, la evaluación para el mejoramiento de procesos es un eje importante para que se considere eficiente la atención, de manera tal que los pacientes sientan satisfechas sus necesidades físicas y emocionales.

Lo encontrado en los resultados por parte de los investigadores logra definir que la percepción favorable que tienen los pacientes acerca de los cuidados e intervenciones brindados por parte del profesional de enfermería, fortalece la interacción enfermero(a)-paciente, aumenta la seguridad en la atención y contribuye al mejoramiento de la calidad del cuidado de enfermería.

\section{Conclusiones}

- De acuerdo con el instrumento Care-Q, la calidad del cuidado de enfermería encontrada en los pacientes con tratamiento de hemodiálisis es buena, según los resultados obtenidos en la investigación: en las seis subescalas predomino el sentirse muy satisfecho con porcentajes entre $60 \%$ y $85 \%$.

- Dentro de la caracterización demográfica se encontró que la mayor parte de la población pertenece al régimen subsidiado y a los estratos 1 , 2 y 3 . Sin embargo, estas condiciones no afectaron el concepto favorable que tienen los pacientes acerca de la prestación del servicio y, en especial, el cuidado brindado por el enfermero(a).

- La subescala de Monitorea y hace seguimiento representó la mayor satisfacción percibida por los pacientes, soportada con actividades como la organización que demuestra el profesional de enfermería en la realización de actividades, la seguridad y tranquilidad que trasmite el enfermero(a) y la realización oportuna y eficiente de procedimientos e intervenciones.

- Durante la realización del estudio se hizo evidente el papel fundamental de enfermería en el cuidado de los pacientes con tratamiento de hemodiálisis. En los resultados se encontró un
E-ISSN 2322-7028

Vol. 15 No. 1

Ene - Jun 2018

Cúcuta, Colombia 
ISSN-PRINT

1794-9831

E-ISSN 2322-7028

Vol. 15 No. 1

Ene - Jun 2018

Cúcuta, Colombia porcentaje considerable de satisfacción dentro de la población participante, lo cual se contrasta con lo evidenciado en la literatura, donde se plantea que el enfermero(a) es el profesional más indicado para el cuidado de este tipo de paciente.

- El presente estudio fue fundamental para evidenciar la necesidad de generar investigaciones dirigidas a la evaluación continua del cuidado de enfermería, debido a que permite la creación de acciones de mejoramiento de la gestión del cuidado, siendo ésta una de las estrategias efectivas para garantizar el cumplimiento de la calidad y para la instauración de diagnósticos situacionales en las organizaciones de salud.

\section{Conflicto de intereses}

Los autores declaran no tener ningún conflicto de intereses.

\section{Referencias Bibliográficas}

1. Torres Saumeth KM, Ruiz Afanador TS, Solís Ospino L, Martínez Barraza F. Calidad y su evolución: una revisión. Dimens. empres. 2012; 10(2):100-107.

2. Puch Ku GF, Uicab Pool GA, Ruiz Rodríguez M, Castañeda Hidalgo H. Dimensiones del cuidado de enfermería y la satisfacción del paciente adulto hospitalizado. Rev Enferm Inst Mex Seguro Soc. 2016;24(2):123-8

3. Coronado Zarco R, Cruz Medina E, Macías HSI, Arellano HA, Nava BT. El contexto actual de la calidad en salud y sus indicadores. Rev Mex Med Fis Rehab. 2013; 25(1):26-33.

4. Rodríguez Jiménez, S, Cárdenas Jiménez, M, Pacheco Arce, A, Ramírez Pérez, M. Una mirada fenomenológica del cuidado de enfermería. Enfermería Universitaria. 2014; 11(4):145-153.

5. Rodríguez Martínez R. Modelos de enfermería integrados al cuidado del paciente nefrológico en el contexto cubano. Rev Cubana Enfermer. 2012; 28(4):474-484.

6. Calabia ER, Piñera Haces C, Izquierdo M, Arias Rodríguez MA. Insuficiencia renal aguda (I). Concepto. Epidemiología. Clasificación. Etiopatogenia. Indicadores de gravedad. [internet] 2007. [Consultado 22 febrero 2017]. Disponible en: https://dialnet.unirioja.es/servlet/articulo? codigo $=2322276$

7. Ministerio de Salud y Protección Social. Programa Nacional de Salud Renal [internet]. [Consultado: 2016 febrero 22]. Disponible en: https://www.minsalud.gov.co/sites/rid/Lists/BibliotecaDigital/RIDE/ INEC/CAC/programas_ERC.pdf

8. Acuña L, Sánchez P, Soler LA, Alvis LF. Enfermedad renal crónica en Colombia: prioridad para la gestión de riesgo. Rev Panam Salud Pública. 2016; 40(1):16-22.

9. Lopera Medina, MM. La enfermedad renal crónica en Colombia: necesidades en salud y respuesta del Sistema General de Seguridad Social en Salud. Revista Gerencia y Políticas de Salud. 2016;15(30):212-233

10. Ministerio de Salud y Protección Social - Ministerio de Hacienda y Crédito Público. Cuenta de alto costo - Enfermedad Renal Crónica. [internet]. [Consultado: 2016 febrero 22]. Disponible en: https:// cuentadealtocosto.org/site/index.php/patologias/9-patologias/35-enfermedad-renal-cronica-erc/

11. Chávez D, Romeo R, Zúñiga J. Percepción de la calidad del cuidado de enfermería en pacientes hospitalizados en el Hospital Universitario del Caribe. [Tesis pregrado]. Cartagena de Indias D.T y C: Universidad de Cartagena. Facultad de Enfermería. [internet]. [Consultado: 2017 enero 27]. Disponible en: http://190.242.62.234:8080/jspui/bitstream/11227/2686/1/informefinal.pdf

12. Rosales Origuela J, Díaz Díaz J, Molina Ramírez B, Chávez Troya O. Ética en los cuidados de enfermería a pacientes en tratamiento con hemodiálisis. Medisur. 2016; 14(5):512-515.

13. Ortíz Z AC. Dimensión del servicio de enfermería. Investigación y Educación en Enfermería. 2003; 21(1):86-95.

14. Larson PJ. Important nurse caring behaviors perceived by patients with cancer. Oncol Nurs Forum.1984; 11(6):46-50.

15. Sepúlveda Carrillo GJ, Rojas Cifuentes LA, Cárdenas Jiménez OL, Guevara Rojas E, Castro Herrera AM. Estudio piloto de la validación del cuestionario "Care-Q" en versión al español en población colombiana. Revista Colombiana de Enfermería. 2009;4(4):13-22 
16. Johnston CB. Patient Satisfaction and Its Discontents. JAMA Intern Med. 2013; 173(22):2025-2026.

17. Sorra J, Khanna K, Dyer N, Mardon R, Famolaro T. Exploring relationships between patient safety culture and patients' assessments of hospital care. J Patient Saf. 2012; 8(3):131-139.

18. Anhang PR, Elliott MN, Zaslavsky AM. Valuing Patient Experience as a Unique and Intrinsically Important Aspect of Health Care Quality. JAMA Surg. 2013; 148(10):985-986.

19. Rojas Martínez W, Barajas Lizarazo MA. Percepción de la calidad del cuidado de enfermería en el servicio de urgencias. Revista Ciencia y Cuidado. 2016; 9(1):13-23.

20. Lyu H, Cooper M, Freischlag JA, Makary MA. Patient Satisfaction as a Possible Indicator of Quality Surgical Care-Reply. JAMA Surg. 2013;148(10):986-987

21. Pantouvakis A, Bouranta N. Quality and price-impact on patient satisfaction. International Journal of Health Care Quality Assurance. 2014; 27(8): 684-696.

22. Daiker B. The patient experience: mining the data to improve your score. Minn Med. 2014; 97(8):38-40.

23. Ahmed F, Burt J, Roland M. Measuring patient experience: concepts and methods. Patient. 2014; 7(3): 235-41.

24. Noest S, Ludt S, Klingenberg A, Glassen K, Heiss F, et al. Involving patients in detecting quality gaps in a fragmented healthcare system: development of a questionnaire for Patients> Experiences Across Health Care Sectors (PEACS). Int J Qual Health Care, 2014; 26(3):240-9.

25. Machuca Albarracín DA. Calidad o excelencia "una preocupación de la gerencia". Revista Ciencia y Cuidado. 2017; 3(1):140-146.

26. Moreno Fergusson ME. Editorial: El fenómeno de la cronicidad: su impacto en el individuo, en su familia y el cuidado de enfermería. Aquichan. 2014; 14(4):458-59.

27. Barrera L, Carrillo GM, Chaparro L, Pinto N, Rodriguez A, Sánchez B. Effect of the program "caring for caretakers": findings of a multicenter study. Colombia Médica. 2011; 42(2):35-44.

28. Cortés D, Serrezuela Tamayo AJ. Nivel de satisfacción de los pacientes que asisten al servicio de urgencias, frente a la atención de enfermería en una institución de cuarto nivel de atención en salud en Bogotá. Septiembre-Octubre de 2008. [Tesis pregrado]. Bogotá: Pontificia Universidad Javeriana. Facultad de Enfermería. [internet]. [Consultado: 2017 Octubre 3]. Disponible en: https://repository. javeriana.edu.co/bitstream/handle/10554/9783/Tesis12.pdf?sequence=1

29. Mesa Estrada H, Orellano Ruiz Y, Varela Polo A, Agudelo Chona M. Satisfacción de los pacientes postquirúrgicos frente al cuidado de Enfermería Hospitales Niño Jesús, ESE CARI alta complejidad y Universidad del Norte Barranquilla Marzo-Mayo de 2010. [Tesis pregrado]. Barranquilla: Universidad del Norte. Facultad de Enfermería. Barranquilla. [internet]. [Consultado: 2017 Octubre 3]. Disponible en:http:// manglar.uninorte.edu.co/bitstream/handle/10584/5251/Satisfacciondelospacientes.pdf?sequence=1

30. Molina Cardona EM. Evaluación de la calidad del cuidado de enfermería en un servicio de hospitalización domiciliaria en Bogotá D.C. [Tesis Maestría]. Bogotá: Universidad Nacional de Colombia. Facultad de Enfermería. 2011. Bogotá, Colombia. [internet]. [Consultado: 2017 Octubre 3]. Disponible en: http://www.bdigital.unal.edu.co/6471/1/Edithmilenamolinacardona.2011.pdf

31. Bautista R., LM. Percepción de la calidad del cuidado de enfermería en la ESE Francisco de Paula Santander. Aquichan. 2008; 8(1):74-84.

32. Appleby C, Camacho BR. Retos y oportunidades: aportaciones de la Enfermera de Práctica Avanzada en la cronicidad. Aprendiendo de las experiencias. Enfermería Clínica. 2014; 24(1):90-98.

\section{Bibliografía}

1. Ángel Ángel ZE, Duque Castaño GA, Tovar Cortes DL. Cuidados de enfermería en el paciente con enfermedad renal crónica en hemodiálisis: una revisión sistemática. Enferm Nefrol. 2016; 19(3):202-213.

2. Aguayo Acuña F, Mella Moraga R. Significado práctico del concepto gestión del cuidado en las enfermeras/os que se desempeñan en atención primaria en salud. Cienc. enferm. 2015; 21(3):73-85.

3. Báez Hernández FJ, Nava Navarro V, Ramos Cedeño L, Medina López O. El significado de cuidado en la práctica profesional de enfermería. Aquichán. 2009; 9(2):127-134.

4. Barbosa V, Alvarado O. Enfermería una disciplina social. Enfermería en Costa Rica. 2011; 32(1):81-88.

5. Barros Higgins L, Herazo Beltrán Y, Aroca Martínez G. Calidad de vida relacionada con la salud en 
ISSN-PRINT

$1794-9831$

E-ISSN 2322-7028

Vol. 15 No. 1

Ene - Jun 2018

Cúcuta, Colombia

pacientes con enfermedad renal crónica. rev.fac.med. 2015; 63(4):641-647.

6. Bautista Rodríguez LM, Arias Velandia MF, Carreño Leiva ZO. Percepción de los familiares de pacientes críticos hospitalizados respecto a la comunicación y apoyo emocional. Rev Cuid. 2016; 7 (2):1297-1309.

7. Borré Ortiz YM, Vega Vega Y. Calidad percibida de la atención de enfermería por pacientes hospitalizados. Cienc. enferm. 2014; 20(3):81-94.

8. Durán de Villalobos MM. Marco epistemológico de la enfermería. Aquichán. 2002; 2(1):7-18.

9. Freitas Santana J, Silva Bauer AE, Minamisava R, Bezerra Queiroz AL, Sousa Maiana RG. Calidad de los cuidados de enfermería y satisfacción del paciente atendido en un hospital de enseñanza. Rev. Latino Am. Enfermagem. 2014; 22(3):454-460.

10. García Hernández, MdL, Cárdenas Becerril, L, Arana Gómez, B, Monroy Rojas, A, Hernández Ortega, Y, Salvador Martínez, C. Construcción emergente del concepto: cuidado profesional de enfermería. Texto \& Contexto Enfermagem. 2011; 20:74-80.

11. Gómez Rodríguez M, Moreno Olivencia P, Miralles Martínez F, Sánchez Martos MD, Sánchez Lamolda MA, Pérez Molina G. Cuidados de calidad y satisfacción del paciente con enfermería en hemodiálisis. Enferm Nefrol. 2014; 17(Suppl 1):94-94.

12. Guerra de guerrero V, Sanhueza Alvarado O, Cáceres Espina M. Calidad de vida de personas en hemodiálisis crónica: relación con variables sociodemográficas, médico-clínicas y de laboratorio. Rev. Latino-Am. Enfermería. 2012; 20(5):838-846.

13. Lopera Medina, MM. La enfermedad renal crónica en Colombia: necesidades en salud y respuesta del Sistema General de Seguridad Social en Salud. Revista Gerencia y Políticas de Salud. 2016; 15(30):212-233.

14. Ministerio de $\quad$ Educación. $\quad$ Ley 911 de 2004 por la cual se dictan disposiciones en materia de responsabilidad deontológica para el ejercicio dela profesión de Enfermería en Colombia; se establece el régimen disciplinario correspondiente y se dictan otras disposiciones. (Octubre 5 de 2004).

15. Olivera Jeréz JM, de la Rúa Méndez E, Gracia Nicado A, Ramírez Olivera AM. Percepción de riesgo del enfermero en el cuidado del paciente viviendo con VIH-sida. Rev Cubana Enfermer. 2012; 28(4):521-531.

16. Pisano M, González A. González P. La modificación de los hábitos y la adherencia terapéutica, clave para el control de la enfermedad crónica. Enferm. Clín. 2014;24(1):59-66

17. Ramírez Estrada, MC, Gil Ospina, AA. Percepción de calidad en servicios de urgencias hospitalarias de II nivel, Risaralda Colombia, 2013. Investigaciones Andina. 2016; 18(32):1507-1520.

18. Rico Landazábal A, Perea D, Garizabalo O, Sanabria M, Vesga J, Ronderos I, et al. Programa de prevención de la enfermedad renal crónica basado en redes integradas de servicios en Colombia. Rev. salud pública. 2017; 19(2):171-176.

19. Rodríguez Jiménez, S, Cárdenas Jiménez, M, Pacheco Arce, A, Ramírez Pérez, M. Una mirada fenomenológica del cuidado de enfermería. Enfermería Universitaria. 2014; 11(4):145-153.

20. Rodríguez Martínez R. Modelos de enfermería integrados al cuidado del paciente nefrológico en el contexto cubano. Rev Cubana Enfermer. 2012; 28(4):474-484.

21. Sepúlveda Carrillo GJ, Rojas Cifuentes LA, Cárdenas Jiménez OL, Guevara Rojas E, Castro Herrera AM. Estudio piloto de la validación del cuestionario "Care-Q" en versión al español en población colombiana. Revista Colombiana de Enfermería. 2009;4(4):13-22

22. Soto Arreola M. El reto de la calidad y seguridad de la atención de enfermería. Revista Conamed. 2013; 18(1):53-55.

23. Torres C, Páez A, Rincón L, Rosas D, Mendoza E. Reproducibilidad del cuestionario: calidad de cuidados de enfermería en pacientes hospitalizados. Revista Cuidarte. 2016; 7(2):1338-1344.

24. Torres Saumeth K, Ruiz Afanador T, Solís Ospino L, Martínez Barraza F. Calidad y su evolución: una revisión. Dimens. Empres. 2012; 10(2):100-107. 